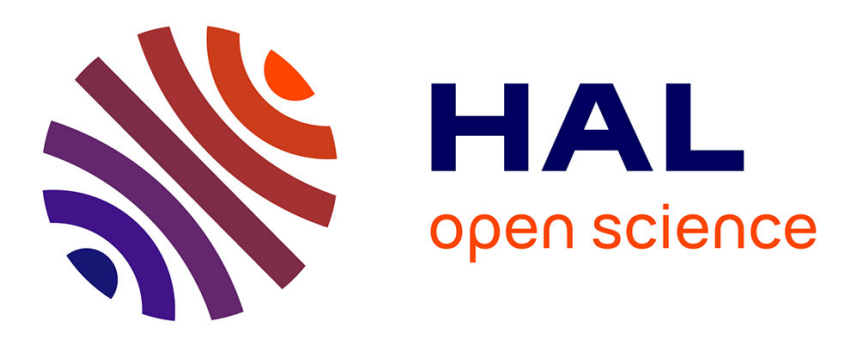

\title{
Discrete numerical analysis of failure modes in granular materials
}

\author{
Luc Sibille, Florent Prunier, François Nicot, Félix Darve
}

\section{To cite this version:}

Luc Sibille, Florent Prunier, François Nicot, Félix Darve. Discrete numerical analysis of failure modes in granular materials. Computational Methods in Applied Sciences, 25, Springer, pp.187-210, 2011, Particle-Based Methods, 10.1007/978-94-007-0735-1_7 . hal-01007503

\section{HAL Id: hal-01007503 https://hal.science/hal-01007503}

Submitted on 4 Feb 2018

HAL is a multi-disciplinary open access archive for the deposit and dissemination of scientific research documents, whether they are published or not. The documents may come from teaching and research institutions in France or abroad, or from public or private research centers.
L'archive ouverte pluridisciplinaire HAL, est destinée au dépôt et à la diffusion de documents scientifiques de niveau recherche, publiés ou non, émanant des établissements d'enseignement et de recherche français ou étrangers, des laboratoires publics ou privés. 


\title{
Discrete Numerical Analysis of Failure Modes in Granular Materials
}

\author{
Luc Sibille \\ Laboratoire GeM - Université de Nantes, ECN, CNRS - IUT de St-Nazaire, BP 420, \\ 44606 St-Nazaire Cedex, France; e-mail: luc.sibille@univ-nantes.fr \\ Florent Prunier \\ LGCIE, INSA-Lyon, Université de Lyon, 69621 Villeurbanne, France; \\ e-mail: florent.prunier@insa-lyon.fr \\ François Nicot \\ Unité ETNA, Cemagref de Grenoble, BP 76, 38402 St-Martin-d'Hères Cedex, France; \\ e-mail: francois.nicot@cemagref.fr \\ Félix Darve \\ Laboratoire 3S-R - INPG, UJF, CNRS, BP 53, 38041 Grenoble Cedex 9, France; \\ e-mail: felix.darve@inpg.fr
}

\begin{abstract}
The question of failure for geomaterials, and more generally for nonassociative materials, is revisited through the second-order work criterion defining, for such media, a whole domain of bifurcation included in the plastic limit surface. In a first theoretical part of the chapter, relations between the vanishing of the second-order work, the existence of limit states and the occurrence of failures characterized by a transition from a quasi-static pre-failure regime to a dynamic post-failure regime, are presented and illustrated from discrete element computations. Then boundaries of the bifurcation domain and cones of unstable loading directions are given in fully three-dimensional loading conditions for a phenomenological incrementally non-linear relation, and in axisymmetric loading conditions for a numerical discrete element model. Finally, conditions for the triggering and the development of failure inside the bifurcation domain are described and emphasized from direct simulations with the discrete element method for proportional stress loading paths.
\end{abstract}




\section{Introduction}

The question of failure is central in engineering and it has been tackled from theoretical, experimental and numerical points of view. This question is delicate, particularly for geomaterials (soils, rocks, concretes) because of the non-associative character of their plastic strains, which implies the existence of a whole domain of bifurcations in the stress space and not only a single plastic limit surface concentrating all kinds of failures as for associative materials.

From a theoretical point of view the existence of such a bifurcation domain was clearly established since the works by Hill [9]. Indeed, roughly speaking, a nonassociative material has a non-symmetrical elasto-plastic matrix. Thus the secondorder work criterion, linked to vanishing values of the determinant of the symmetric part of this constitutive matrix, can be satisfied before the plastic limit condition, linked to vanishing values of the determinant of the constitutive matrix itself. The existence of failure states before the plastic limit has been also proven by Rice [16] since for a non-associative material the determinant of the acoustic tensor (criterion for shear band formation) can vanish in the hardening regime (i.e. before the plastic limit condition). In the first part of this paper, the theoretical framework is recalled by emphasizing the link between second-order work, kinetic energy and limit states. Indeed failure is due to the existence of some limit states (classically limit stress states). Besides, failure is associated to some bursts of kinetic energy at the transition from the quasi-static pre-failure regime to the dynamic post-failure regime. Thus, the first part is devoted to clarify the link between these three notions. Then a phenomenological elasto-plastic relation (an incrementally non-linear model) applied to 3D loading conditions allows to show the boundary of the bifurcation domain in 3D and the instability cones, where the second-order work is taking negative values.

From an experimental point of view, some failure states strictly inside MohrCoulomb's surface have been observed since many years typically for given loading paths. The most classical ones giving rise to a diffuse mode of failure are the undrained triaxial compressions on a loose sand for axially force controlled tests and the drained triaxial tests with a constant deviatoric stress for a constant injection rate of water inside the sample (see, for example, [5]). Localized failures have been also observed repeatedly experimentally in the hardening regime on dense sands [6].

From a numerical viewpoint, the modelling of failure modes with a numerical investigation of all the mechanical/geometrical details is a difficult task with the finite element method, essentially because such a numerical method is typically not well adapted to the description of bifurcation states. This is the reason why this paper is devoted to a numerical investigation of failure inside granular materials through a discrete element method. This method has indeed the great advantage to simulate failure in a very realistic and natural way. Thus, the main part of this paper is devoted to the analysis of failure inside a cubical specimen of 10,000 spheres. It will be observed first that, in close agreement with experiments and theory, there exists a stress bifurcation domain strictly inside Mohr-Coulomb's surface and that some instability cones gather the stress directions where the second-order work is taking 
negative values. Besides, elasto-plastic theory shows that there are three necessary and sufficient conditions for an effective failure:

1. the stress state has to be inside the bifurcation domain,

2. the loading direction must belong to the current instability cone,

3. the (energy conjugate) loading variables must be mixed ones (i.e. some stresses and strains).

The capacity of discrete element method to check these three necessary and sufficient conditions and to simulate all the features of diffuse failure (exponentially growing strains, burst of kinetic energy, decreasing intergranular stresses, not any localization pattern) will be remarkably illustrated in the last part of the chapter.

\section{Theoretical Background}

Considering a soil specimen, failure can be localized (the kinematic field experience a discontinuous aspect) or diffuse (no localization pattern is visible). In this chapter, we focus on the diffuse failure of soil specimens, related to an exponential increase in strain rates, with outbursts in kinetic energy. That corresponds to the transition from a quasi-static regime (the system reaches an equilibrium state under the external loading) toward a dynamic regime (the internal stress inside the system can no longer balance the external loading, leading to dynamic effects).

Thus, this section investigates in which conditions kinetic energy of a soil specimen, initially in equilibrium after a given loading path, may increase (passing from zero to a strictly positive value) over an infinitesimal loading.

\subsection{Kinetic Energy and Second-Order Work}

For this purpose, let us consider a system of volume $V_{o}$, initially in a configuration $C_{o}$. After a loading history, the system is in a strained configuration $C$ and occupies a volume $V$, in equilibrium under a prescribed external loading. An external stress distribution $f$ acts on the current boundary $(\Gamma)$ of the material.

The instantaneous change in the system, in the equilibrium configuration $C$ at time $t$, is governed by the following energy conservation equation that includes dynamic effects:

$$
\delta E_{c}(t)=\int_{\Gamma} f_{i} \delta u_{i} d S-\int_{V} \sigma_{i j} \frac{\partial\left(\delta u_{i}\right)}{\partial x_{j}} d V
$$

where $\delta E_{c}$ represents the system's current change in kinetic energy related to the incremental displacement field $\delta u$, and $\overline{\bar{\sigma}}$ is the Cauchy stress tensor. Equation (1) represents the Eulerian form of the energy conservation, since all variables are given 
with respect to the current evolving configuration. The notation $\delta X$ represents the incremental change of any variable $X$, equal to $\dot{X} \delta t$.

It is of course more convenient to come back to the initial configuration $C_{o}$, which is fixed. This transformation can be operated on both integrals of Eq. (1), leading to the Lagrangian form of energy conservation [11]:

$$
\delta E_{c}(t)=\int_{\Gamma_{o}} F_{i} \delta u_{i} d S_{o}-\int_{V_{o}} \Pi_{i j} \frac{\partial\left(\delta u_{i}\right)}{\partial X_{j}} d V_{o}
$$

where $\overline{\bar{\Pi}}$ denotes the Piola-Kirchoff stress tensor of the first type and $\Gamma_{o}$ is the $V_{o}$ boundary. $\overline{\bar{\Pi}}$ and $F$ are, respectively, the transformed quantities of $\overline{\bar{\sigma}}$ and $f$ through the bijection mapping the material points from the current configuration to the reference configuration.

Time differentiation of Eq. (2) gives, after some algebra [10]:

$$
2 E_{c}(t+\delta t)=\int_{\Gamma_{o}} \delta F_{i} \delta u_{i} d S_{o}-\int_{V_{o}} \delta \Pi_{i j} \frac{\partial\left(\delta u_{i}\right)}{\partial X_{j}} d V_{o}
$$

Following Hill's definition [9],

$$
W_{2}=\int_{V_{o}} \delta \Pi_{i j} \frac{\partial\left(\delta u_{i}\right)}{\partial X_{j}} d V_{o}
$$

denotes the global second-order work of the system, associated with the incremental change $\left(\delta \Pi_{i j}, \delta\left(\partial u_{i} / \partial X_{j}\right)\right)$. Both incremental quantities $\delta\left(\partial u_{i} / \partial X_{j}\right)$ and $\delta \Pi_{i j}$ are related through the constitutive equation.

In Eq. (3), the boundary integral $\int_{\Gamma_{o}} \delta F_{i} \delta u_{i} d S_{o}$ represents the external loading applied to the system, through the control parameters. In case of a constant loading (or "dead forces", after Hill), the boundary integral $\int_{\Gamma_{o}} \delta F_{i} \delta u_{i} d S_{o}$ vanishes, and Eq. (3) reads:

$$
2 E_{c}(t+\delta t)=-W_{2}
$$

Thus, the occurrence of outburst in kinetic energy is directly related to the vanishing of the second-order work.

Assuming that geometrical changes can be omitted over the incremental evolution considered, (4) can be rewritten as follows:

$$
W_{2}=\int_{V_{o}} \delta \sigma_{i} \delta \varepsilon_{i} d V_{o}=\int_{V_{o}} w_{2} d V_{o}
$$

Moreover if we restrict henceforth the analysis to the context of the material point scale, the second-order work is a quadratic form associated with the symmetric part $\overline{\bar{K}} s$ of the tangent stiffness matrix:

$$
w_{2}=\delta \sigma_{i} \delta \varepsilon_{i}=K_{i j}^{s} \delta \varepsilon_{i} \delta \varepsilon_{j}
$$


Vectorial notations are used in Eq. $(6)$, where $(3 \times 3)$ tensors $\overline{\bar{\sigma}}$ and $\overline{\bar{\varepsilon}}$ were replaced with six components vectors $\sigma$ and $\varepsilon$.

Equation (7) shows that the second-order work varies with the direction of $\delta \varepsilon$. Because of its symmetry, all eigenvalues of $\overline{\bar{K}} s$ are real. When the eigenvalues are strictly positive, $w_{2}$ is strictly positive for any direction of $\delta \varepsilon$. $w_{2}$ first vanishes in the same time as $\overline{\bar{K}}^{s}$ admits a nil eigenvalue (in that case, det $\overline{\bar{K}}^{s}=0$ ). As a consequence, the existence of outburst in kinetic energy is strongly related to the spectral properties of $\overline{\bar{K}} s$.

In homogeneous laboratory tests, some components (or linear combinations of components) of strain and stress are imposed, and the response is computed by means of the conjugate variables. Let us exemplify in two-dimensional conditions, by considering proportional strain paths. The incremental axial strain is constant, and both incremental lateral and axial strains are proportional:

$$
\lambda \delta \varepsilon_{1}+\delta \varepsilon_{2}=0 \quad \text { and } \quad \delta \varepsilon_{1}=\text { const. }
$$

To investigate the response along this loading path, the variation of the variable $\delta \sigma_{1}-\lambda \delta \sigma_{2}$ in terms of $\varepsilon_{1}$ is analyzed. Starting from the constitutive relation:

$$
\left[\begin{array}{l}
\delta \sigma_{1} \\
\delta \sigma_{2}
\end{array}\right]=\overline{\bar{K}}\left[\begin{array}{l}
\delta \varepsilon_{1} \\
\delta \varepsilon_{2}
\end{array}\right]
$$

it can be shown that:

$$
\delta \sigma_{1}-\lambda \delta \sigma_{2}=\left(K_{11}-\lambda\left(K_{12}+K_{21}\right)+\lambda^{2} K_{22}\right) \delta \varepsilon_{1}
$$

Noting that:

$$
K_{11}-\lambda\left(K_{12}+K_{21}\right)+\lambda^{2} K_{22}=[1-\lambda]\left[\begin{array}{ll}
K_{11} & K_{12} \\
K_{21} & K_{22}
\end{array}\right]\left[\begin{array}{c}
1 \\
-\lambda
\end{array}\right]
$$

the second-order work $w_{2}(\lambda)$ associated with the incremental strain direction $\overrightarrow{u_{\varepsilon}}=\left[\begin{array}{c}1 \\ -\lambda\end{array}\right]$ reads:

$$
w_{2}(\lambda)=\left(K_{11}-\lambda\left(K_{12}+K_{21}\right)+\lambda^{2} K_{22}\right)\left(\delta \varepsilon_{1}\right)^{2}
$$

Finally, it follows that

$$
\delta \sigma_{1}-\lambda \delta \sigma_{2}=\frac{w_{2}(\lambda)}{\delta \varepsilon_{1}}
$$

When $w_{2}(\lambda)=0$, then $\delta\left(\sigma_{1}-\lambda \sigma_{2}\right)=0$.

As a consequence, the vanishing of the second-order work along the incremental strain direction $\overrightarrow{u_{\varepsilon}}=\left[\begin{array}{c}1 \\ -\lambda\end{array}\right]$ is related to the existence of a maximum for the curve giving the evolution of the variable $\sigma_{1}-\lambda \sigma_{2}$ in terms of $\varepsilon_{1}$. The maximum of $\sigma_{1}-\lambda \sigma_{2}$ corresponds to a proper limit state. 
This is the generalization of the plastic limit condition, observed for example at the peak of the axial stress, for drained triaxial tests. As the lateral stress is assigned to remain constant $\left(\delta \sigma_{2}=0\right)$, the second-order work is vanishing at the axial stress peak: $\delta \sigma_{1}=0$ implying that $\delta \sigma_{1} \delta \varepsilon_{1}+\delta \sigma_{2} \delta \varepsilon_{2}=0$.

The plastic limit condition is a particular example of limit state since both incremental stress components are nil. In the proportional strain loading path discussed above, one incremental stress term $\left(\sigma_{1}-\lambda \sigma_{2}\right)$ passes through a maximum, whereas a strain term $\left(\lambda \varepsilon_{1}+\varepsilon_{2}\right)$ is imposed constant: this is a mixed condition. If the loading is defined with only strain terms, the kinematics on the boundary of the specimen is prescribed, preventing any outburst in kinetic energy from occurring. No failure is therefore visible.

In conclusion, the occurrence of failure mode requires three conditions:

1. One eigenvalue of $\overline{\bar{K}}^{s}$ is negative or nil (the mechanical state belongs to the bifurcation domain [4]).

2. The incremental loading direction considered is associated with a negative or nil value of the second-order work.

3. A mixed loading condition has to be applied, involving at least one stress term.

\subsection{DEM Investigation for Proportional Strain Loading Paths}

The two-dimensional example discussed above considering proportional strain paths, is illustrated here through numerical experiments based on the discrete element method (DEM) [1]. However, the granular assembly considered below constitutes a three-dimensional sample loaded in axisymmetric conditions with respect to direction ' 1 ' $\left(\sigma_{2}=\sigma_{3}\right.$ and $\left.\varepsilon_{2}=\varepsilon_{3}\right)$. In these conditions, the proportional strain loading defined in Eq. (8) writes:

$$
\lambda \delta \varepsilon_{1}+2 \delta \varepsilon_{3}=0 \quad \text { and } \quad \delta \varepsilon_{1}=\text { positive const. }
$$

The granular assembly considered has a cubical shape and is composed of about 10,000 spheres [19]. The inter-particle interaction at contact points is modeled, in the normal direction to the tangent contact plane, by a purely elastic behaviour (characterized by a stiffness $k_{n}$ ). For the direction included in the tangent contact plane the relation is elastic perfectly plastic (characterized by a stiffness $k_{t}$ and a friction angle $\varphi_{c}$ ). Simulations were performed with the code SDEC developed by Donzé and Magnier [7]. In the following of this section we consider a dense numerical sample $E 1$ (characteristics are given in Table 1) exhibiting a dilatant behaviour during classical triaxial compressions.

In Figure 1a the change of variable $\sigma_{1}-\lambda \sigma_{3}$ is shown in terms of $\varepsilon_{1}$ for $\lambda=1.21$ and corresponding to the incremental strain direction $\overrightarrow{u_{\varepsilon}}=\left[\begin{array}{c}1 \\ -1.21 \\ -1.21\end{array}\right]$. For this strain direction, the evolution of $\sigma_{1}-\lambda \sigma_{3}$ presents a maximum [5] and the second-order work plotted in Figure 1c vanishes at this maximum as shown by Eq. (13). The 
Table 1 Characteristics of the numerical samples

\begin{tabular}{llllll}
\hline Sample & $\begin{array}{l}k_{n} / d_{s}{ }^{*} \\
(\mathrm{MPa})\end{array}$ & $k_{t} / k_{n}$ & $\begin{array}{l}\varphi_{c} \\
(\mathrm{deg})\end{array}$ & $\begin{array}{l}\text { Void ratio } \\
e\end{array}$ & $\begin{array}{l}\text { Coordination } \\
\text { number } z\end{array}$ \\
\hline$E 1$ & 356 & 0.42 & 35.0 & 0.618 & 4.54 \\
$E 3$ & 356 & 0.42 & 35.0 & 0.693 & 4.42 \\
\hline
\end{tabular}

${ }^{*} d_{s}$ represents the sphere diameter

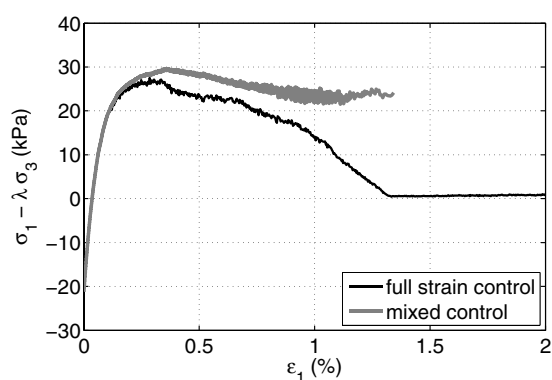

(a)

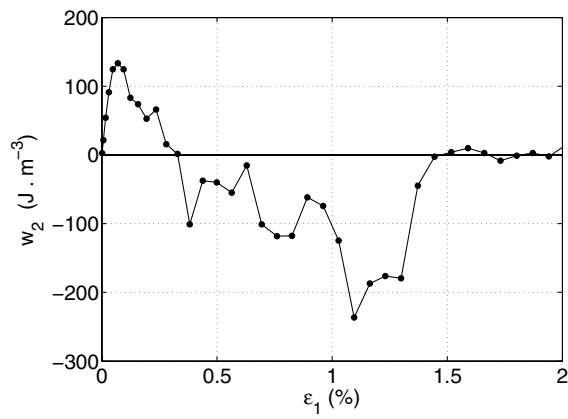

(c)

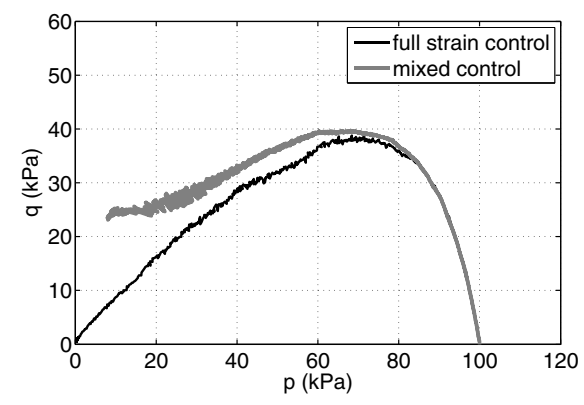

(b)

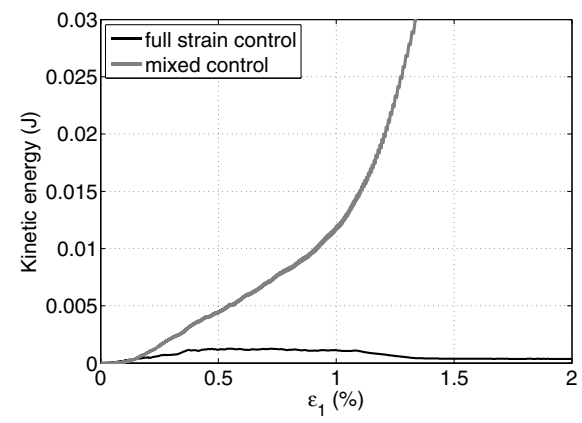

(d)

Fig. 1 (a), (b), (d) Comparison of simulated responses for a proportional strain loading path between a full strain control $\left(\lambda \delta \varepsilon_{1}+2 \delta \varepsilon_{3}=0 ; \delta \varepsilon_{1}>0\right)$ and a mixed control $\left(\lambda \delta \varepsilon_{1}+2 \delta \varepsilon_{3}=0\right.$; $\delta \sigma_{1}-\lambda \delta \sigma_{3}>0$ ); (c) vanishing of the second-order work during the full strain control. $\left(p=\left(\sigma_{1}+2 \sigma_{3}\right) / 3 ; q=\sigma_{1}-\sigma_{3}\right)$

control parameters of the loading are, in this case, defined with only strain terms (Eq. 14), and the kinetic energy of the sample (equal to the sum of the kinetic energy of all particles) stays low $^{1}$ (Figure 1d). The simulation can be carried on until reaching the total quasi-static liquefaction (see the vanishing of stresses in Figure 1b) where the kinetic energy also vanishes.

We consider now the case where the control parameters are mixed and defined by:

\footnotetext{
${ }^{1}$ As the discrete element method is a dynamic method, all evolutions of the granular assembly, even quasi-static, imply production of kinetic energy.
} 


$$
\lambda \delta \varepsilon_{1}+2 \delta \varepsilon_{3}=0 \quad \text { and } \quad \delta \sigma_{1}-\lambda \delta \sigma_{3}=\text { positive const. }
$$

The response of the sample is compared with the previous one in Figures 1a, b and d. The maximum of $\sigma_{1}-\lambda \sigma_{3}$, corresponding to the vanishing of $W_{2}$, cannot be exceeded. Moreover, while the maximum of $\sigma_{1}-\lambda \sigma_{3}$ is approached, an outburst of kinetic energy is developing (as explained by Eq. 5) and sample never gets back to an equilibrium state. This response corresponds to a very sudden failure of the sample. Actually, in Figure 1a, the peak of $\sigma_{1}-\lambda \sigma_{3}$ is slightly exceeded because failure has began to develop slightly before it, with a sharp increase of kinetic energy. Hence the sample response switches from a quasi-static regime to a dynamic regime where contribution of inertial terms in the stress state are not anymore negligible. This dynamic response explains also the non-vanishing of the shear stress $q=\sigma_{1}-\sigma_{3}$ in Figure 1b.

\section{Cones of Unstable Loading Directions, Bifurcation Domain}

\subsection{Basic Concepts}

As seen in relation (7) expression of $w_{2}$ reads

$$
w_{2}=\delta \varepsilon_{i} K_{i j}^{s} \delta \varepsilon_{j}
$$

or equivalently when $\overline{\bar{K}}$ is invertible

$$
w_{2}=\delta \sigma_{i} S_{i j}^{S} \delta \sigma_{j}
$$

with $S=K^{-1}$. Writing the constitutive relation in principal axes in threedimensional conditions:

$$
\left[\begin{array}{l}
\delta \varepsilon_{1} \\
\delta \varepsilon_{2} \\
\delta \varepsilon_{3}
\end{array}\right]=\left[\begin{array}{ccc}
\frac{1}{E_{1}} & -\frac{\nu_{21}}{E_{2}} & -\frac{\nu_{31}}{E_{3}} \\
-\frac{\nu_{12}}{E_{1}} & \frac{1}{E_{2}} & -\frac{\nu_{32}}{E_{3}} \\
-\frac{\nu_{13}}{E_{1}} & -\frac{\nu_{23}}{E_{2}} & \frac{1}{E_{3}}
\end{array}\right]\left[\begin{array}{l}
\delta \sigma_{1} \\
\delta \sigma_{2} \\
\delta \sigma_{3}
\end{array}\right]
$$

Equation (16) can be developed as follows:

$$
\begin{aligned}
& \frac{\delta \sigma_{1}^{2}}{E_{1}}+\frac{\delta \sigma_{2}^{2}}{E_{2}}+\frac{\delta \sigma_{3}^{2}}{E_{3}}-\left(\frac{\nu_{12}}{E_{1}}+\frac{\nu_{21}}{E_{2}}\right) \delta \sigma_{1} \delta \sigma_{2}-\cdots \\
& \left(\frac{\nu_{32}}{E_{3}}+\frac{\nu_{23}}{E_{3}}\right) \delta \sigma_{3} \delta \sigma_{2}-\left(\frac{\nu_{13}}{E_{1}}+\frac{\nu_{31}}{E_{2}}\right) \delta \sigma_{1} \delta \sigma_{3}=0
\end{aligned}
$$

The left-hand side of this equation is a quadric, with neither constant terms nor terms of degree one according to $\delta \sigma_{i}$. Consequently, the solution is an elliptical 


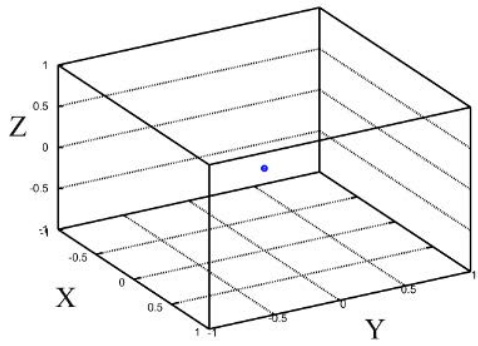

$\alpha_{1}>0, \alpha_{2}>0, \alpha_{3}>0$

(a)

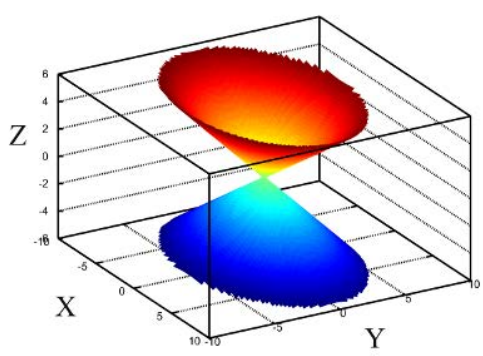

$\alpha_{1}>0, \alpha_{2}>0, \alpha_{3}<0$

(c)

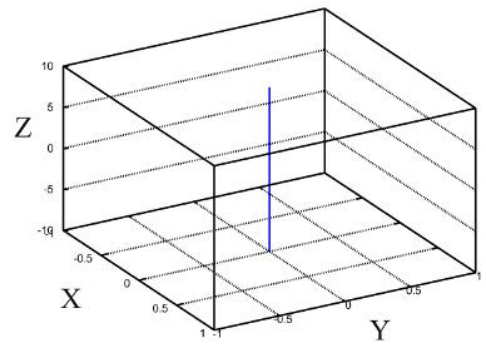

$\alpha_{1}>0, \alpha_{2}>0, \alpha_{3}=0$

(b)

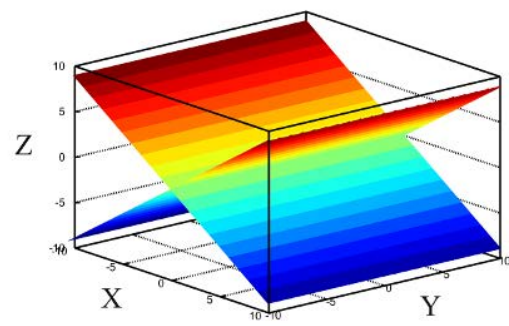

$\alpha_{1}>0, \alpha_{2}=0, \alpha_{3}<0$

(d)

Fig. 2 Solutions of equation: $\alpha_{1} X^{2}+\alpha_{2} Y^{2}+\alpha_{3} Z^{2}=0$

cone if the quadric is not degenerated. The real nature of this solution depends on the positiveness of $\operatorname{det}(\overline{\bar{S}} s)$. By calling $\left(\alpha_{1}, \alpha_{2}, \alpha_{3}\right)$ the eigenvalues of $\overline{\bar{S}}^{s}$, the four possible solutions are displayed in Figure 2.

Through these results, the directional nature of the second-order criterion is established. Nevertheless the previous development holds only for incrementally linear materials that is to say for elastic behaviour. In fact, for elasto-plastic materials the constitutive relation is at least incrementally piece-wise linear with a linear relation in plastic loading regime, and an other linear relation in unloading regime. That is why the above discussion has to be made in a given tensorial zone. ${ }^{2}$ Moreover, it is necessary to verify that solutions belong geometrically to the tensorial zone considered and to cut them (i.e. to keep only the part of the cone include in the tensorial zone for example).

Furthermore, if we make the assumption that eigenvalues of $\overline{\bar{S}} s$ are strictly positive at the virgin state and are evolving continuously with the loading parameter, solutions appear sorted like presented in Figure 2. First, $\operatorname{det}(\overline{\bar{S}} s)>0$, and no nonzero solutions exist. Second $\operatorname{det}\left(\overline{\bar{S}}^{s}\right)=0$, there is only one unstable loading dir-

\footnotetext{
${ }^{2}$ A tensorial zone is a domain of the loading space in which the incremental constitutive relation is linear [3].
} 
ection. Third, $\operatorname{det}(\overline{\bar{S}} s)<0$, an elliptical cone of unstable loading directions appears. Finally, $\operatorname{det}\left(\overline{\bar{S}}^{s}\right)$ might vanish again and unstable loading directions would be included between the intersection of the two planes. Nevertheless we have never observed such solution with the constitutive models used.

Hence it is now possible to define the limit of the bifurcation domain. This limit is the surface gathering all mechanical states for which only one unstable loading direction exist. With the assumptions of positiveness of the eigenvalues at the virgin state and of their continuous evolution with the loading parameter, the limit of the bifurcation domain is given by the following relation for incrementally piece-wise linear model [15]:

$$
\min _{i=1, \ldots, n}\left(\operatorname{det}\left(\overline{\bar{S}}^{s}\right)_{\subset} Z_{i}\right)=0 \quad \text { with } \quad \underline{u_{i}} \subset Z_{i}
$$

with $n$ the number of tensorial zones of the constitutive model, $u_{i}$ the eigenvector corresponding to the vanishing eigenvalue, and $Z_{i}$ the tensorial zone considered.

Eventually, same analysis can be performed in the strain rate space without any restriction. In fact it can be proved that [14]

$$
\operatorname{det}\left(\overline{\bar{K}}^{s}\right)=\frac{\operatorname{det}(\overline{\bar{S}} s)}{(\operatorname{det}(\overline{\bar{S}}))^{2}}
$$

As a consequence both determinant vanish at the same time.

\subsection{Illustration}

We propose now to illustrate remarks made above. Numerical results, are displayed with the constitutive models of Darve [2] and with the discrete element method.

\subsubsection{Phenomenological Constitutive Relations}

Without going into details of Darve's models, we just recall that they are not based on the classical concepts of elasto-plasticity. Decomposition of the strain in an elastic and plastic part is not assumed, and no plastic potentials are defined. According to the first model, the non-linear relation which links strain rate to stress rate is directly described by an incrementally non-linear relation. The second model is a simplification of the first one, and becomes incrementally piece-wise linear with eight tensorial zones. In principal axes, these models are written as follows:

$$
\left[\begin{array}{l}
\delta \varepsilon_{1} \\
\delta \varepsilon_{2} \\
\delta \varepsilon_{3}
\end{array}\right]=\frac{1}{2}\left[N^{+}+N^{-}\right]\left[\begin{array}{l}
\delta \sigma_{1} \\
\delta \sigma_{2} \\
\delta \sigma_{3}
\end{array}\right]+\frac{1}{2\|\underline{\delta \sigma}\|}\left[N^{+}-N^{-}\right]\left[\begin{array}{l}
\delta \sigma_{1}^{2} \\
\delta \sigma_{2}^{2} \\
\delta \sigma_{3}^{2}
\end{array}\right]
$$




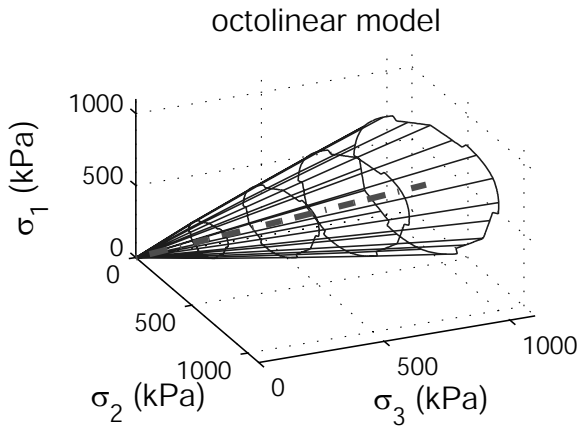

(a)

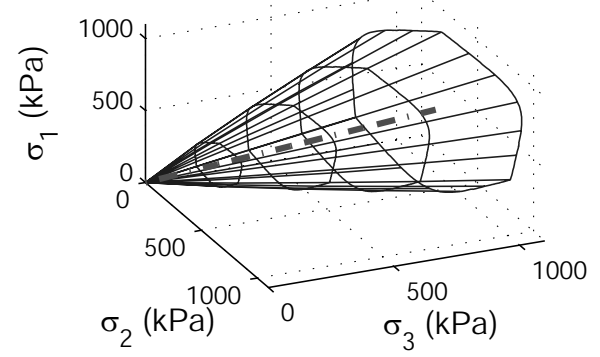

(b)

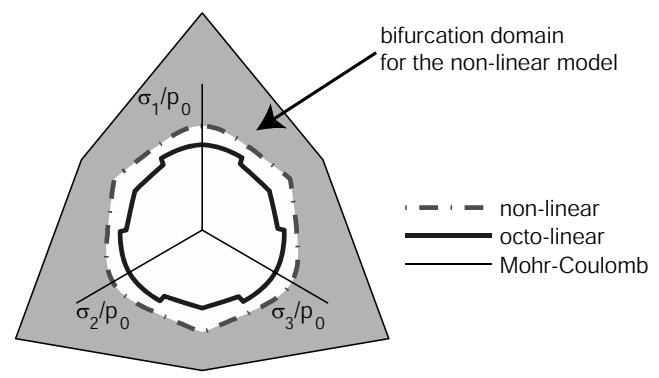

(c)

Fig. 3 Limit of the bifurcation domain plotted in the 3D stress space for constitutive models of Darve

and

$$
\left[\begin{array}{l}
\delta \varepsilon_{1} \\
\delta \varepsilon_{2} \\
\delta \varepsilon_{3}
\end{array}\right]=\frac{1}{2}\left[N^{+}+N^{-}\right]\left[\begin{array}{l}
\delta \sigma_{1} \\
\delta \sigma_{2} \\
\delta \sigma_{3}
\end{array}\right]+\frac{1}{2}\left[N^{+}-N^{-}\right]\left[\begin{array}{l}
\left|\delta \sigma_{1}\right| \\
\left|\delta \sigma_{2}\right| \\
\left|\delta \sigma_{3}\right|
\end{array}\right]
$$

with

$$
N^{ \pm}=\left[\begin{array}{ccc}
\frac{1}{E_{1}^{ \pm}} & -\frac{v_{21}^{ \pm}}{E_{2}^{ \pm}} & -\frac{v_{31}^{ \pm}}{E_{3}^{ \pm}} \\
-\frac{v_{12}^{ \pm}}{E_{1}^{ \pm}} & \frac{1}{E_{2}^{ \pm}} & -\frac{v_{32}^{ \pm}}{E_{3}^{ \pm}} \\
-\frac{v_{13}^{ \pm}}{E_{1}^{ \pm}} & -\frac{v_{23}^{ \pm}}{E_{2}^{ \pm}} & \frac{1}{E_{3}^{ \pm}}
\end{array}\right]
$$

Limits of the bifurcation domain, given by Eq. (17) for the incrementally piecewise linear relation, are displayed in Figure 3 for both models. As a remark, the incrementally non-linear model can be seen as incrementally piece-wise linear with an infinity of tensorial zones. Thus, with a numerical effort, an approximation of the bifurcation limit has been displayed [15]. 


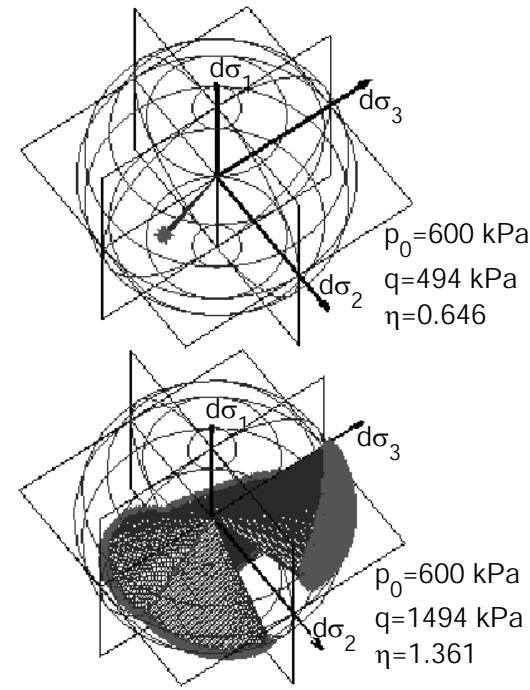

a)

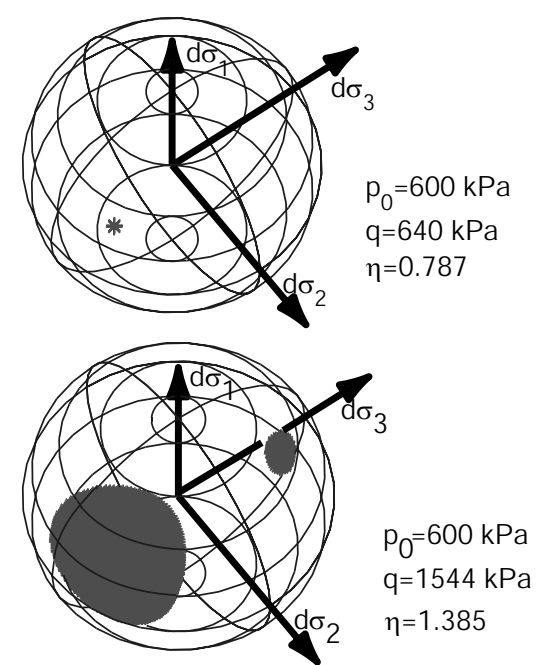

b)

Fig. 4 3D instability cones for a dense sand of Hostun. Figure 4a presents the cones obtained with the octo-linear model. The planes represent the limit between the 8 tensorial zones, the meshes correspond to the analytical solution of Equation (16) and the point clouds the solution obtained with the numerical method. Figure $4 \mathrm{~b}$ shows results obtained with the non-linear model using the numerical method. $p_{o}$ is the initial confining pressure, $q=\sigma_{1}-\sigma_{3}, \eta=q / p$

(a)

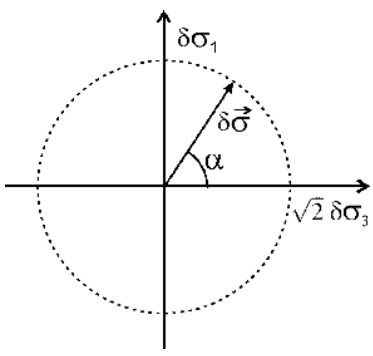

(b)

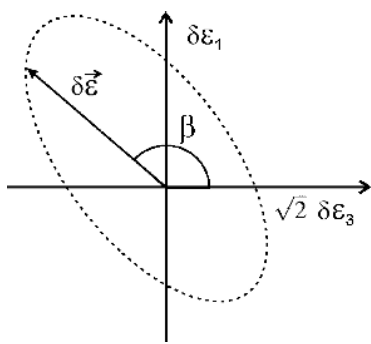

Fig. 5 Definition of stress probes (a), and strain responses (b), in the axisymmetric plane of stress increments and strain increments, respectively

Then, instability cones for stress-strain states situated beyond the bifurcation limit are plotted. Figure 4 presents the 3D cones obtained with Darve's constitutive models [13].

The method used to draw these cones of unstable stress directions consist to realize stress probes [8], as presented in Figure 5a in axisymmetric conditions for simplicity's sake. At different stress states along the loading path (presently a drained triaxial path), a small stress increment $\overrightarrow{\delta \sigma}$, with a constant norm, is applied from 

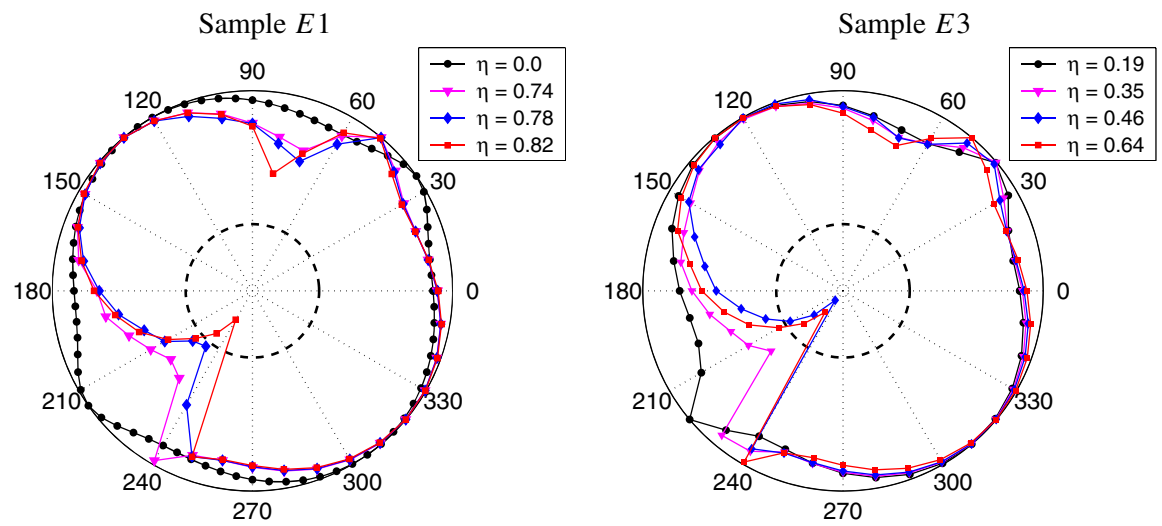

Fig. 6 Circular diagrams of the normalized second-order work at a confinement pressure $\sigma_{3}=$ $100 \mathrm{kPa}$, for samples $E 1$ and $E 3$. The little circle in dash line represent the zero values

the considered stress state, in all directions of the stress increment space. For each stress direction, the corresponding strain response is computed (Figure 5b), giving access to the value of the second-order work. Cones of instability gather, for a given stress state, all stress directions for which $w_{2} \leq 0$. In three-dimensional conditions the principle is the same, but stress probes describe a sphere instead of a circle (Figure 5a).

\subsubsection{Discrete Element Model}

In the same way as with the phenomenological constitutive relations, stress probes have been performed with the DEM along drained triaxial compressions, in axisymmetric conditions only [18]. Two samples are considered here, the dense and dilatant sample $E 1$ (already used for proportional strain loading paths in Section 2.2), and a looser and essentially contractant sample $E 3$ (see characteristics in Table 1). Figure 6 presents circular diagrams of the normalized second-order work $w_{2 n}$ computed from stress probes for a confinement $\sigma_{3}=100 \mathrm{kPa}$ and different deviatoric stress levels $\eta=q / p$. In such diagrams, an arbitrary constant value $c$ is added to the polar value of $w_{2 n}$ in order to have

$$
\forall \alpha, \quad w_{2 n}(\alpha)+c>0,
$$

where $\alpha$ is the stress probe direction (see Figure 5a) and $w_{2 n}$ is defined as

$$
w_{2 n}=\frac{\overrightarrow{\delta \sigma} \cdot \overrightarrow{\delta \varepsilon}}{\|\overrightarrow{\delta \sigma}\|\|\overrightarrow{\delta \varepsilon}\|}
$$

A dashed circle is drawn in the circular diagrams to represent vanishing values of $w_{2 n}$. Outside the dashed circle $w_{2 n}$ is positive, inside it is negative. As for the octo- 

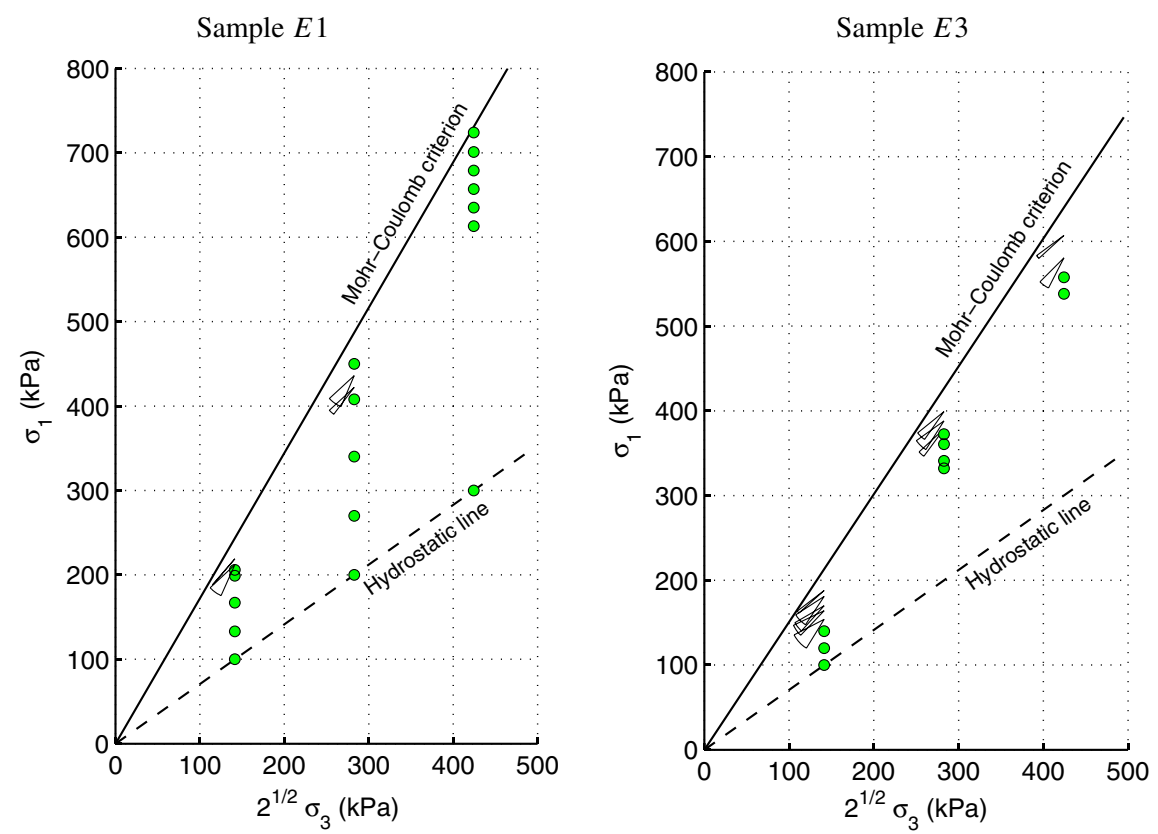

Fig. 7 Synthesis of cones of unstable stress directions in the axisymmetric plane of stresses; full circles represent stress probes for which no vanishing or negative values of $w_{2}$ were found

linear model and the non-linear model (Figure 4), for sufficiently high values of $\eta$ a set of stress directions for which $w_{2} \leq 0$ are found. They form a cone of unstable stress directions. For the loosest sample $E 3$ cones open for much lower deviatoric stress level $\eta$. It is characteristic of a loose sand where the bifurcation domain is wider than for a dense sand, as shown in [4]. As the computational cost to simulate stress probes is quite important, the number of stress probes has been voluntarily limited and we were not able to find the stress state where the first unstable stress direction appears, i.e. the exact limit of the bifurcation domain.

A synthesis of stress probes performed and cones of instability found is presented in Figure 7. One can see a domain strictly included inside the Mohr-Coulomb criterion where cones of instability exist. ${ }^{3}$ This domain constitutes the bifurcation domain and corresponds qualitatively to the bifurcation domain plot in the threedimensional stress space in Figure 3.

\footnotetext{
${ }^{3}$ For the densest sample $E 1$ and the highest confining pressure no cone of instability were found for the tested stress state. This may be due to a compaction of the sample at relatively high pressure, increasing its density and consequently reducing the bifurcation domain [17].
} 


\subsection{Case of the Proportional Strain Loading Path}

In this subsection we consider again proportional strain loading paths presented in Section 2.1 through relation (8). We just extend this description to take into account three-dimensional space as follows:

$$
\begin{cases}\delta \varepsilon_{1}=\text { const. } & \text { const. } \in \mathbb{R}-\{0\} \\ \lambda_{1} \delta \varepsilon_{1}+\delta \varepsilon_{2}=0 & \lambda_{1} \in \mathbb{R} \\ \lambda_{2} \delta \varepsilon_{2}+\delta \varepsilon_{3}=0 & \lambda_{2} \in \mathbb{R}\end{cases}
$$

It can be verified that axisymmetric conditions are obtained with $\lambda_{2}=-1$, plane strain condition with $\lambda_{1}=0$ or $\lambda_{2}=0$, and an undrained axisymmetric triaxial test can be simulated with $\lambda_{2}=-1$ and $\lambda_{1}=1 / 2$.

For such strain path the second-order work reads

$$
\begin{aligned}
w_{2}= & \delta \varepsilon_{1}\left(\delta \sigma_{1}-\lambda_{1} \delta \sigma_{2}+\lambda_{1} \lambda_{2} \delta \sigma_{3}\right)+\left(\lambda_{1} \delta \varepsilon_{1}+\delta \varepsilon_{2}\right)\left(\delta \sigma_{2}-\lambda_{2} \delta \sigma_{3}\right) \\
& +\left(\lambda_{2} \delta \varepsilon_{2}+\delta \varepsilon_{3}\right) \delta \sigma_{3}
\end{aligned}
$$

and consequently, because of condition (24), vanishes at an extremum of $\left(\sigma_{1}-\lambda_{1} \sigma_{2}+\lambda_{1} \lambda_{2} \sigma_{3}\right)$.

To illustrate this, we have considered the following loading program with the incrementally non-linear model. First a drained triaxial path under an initial confining pressure $p_{0}=200 \mathrm{kPa}$ is followed until reaching the state $\sigma_{1}=298 \mathrm{kPa}$, $\sigma_{2}=\sigma_{3}=200 \mathrm{kPa}, \varepsilon_{1}=0.292 \%$ and $\varepsilon_{2}=\varepsilon_{3}=-0.079 \%$. This state is located just before reaching the bifurcation domain limit. From this state, a proportional strain path (24) is applied. In the present case $\lambda_{1}=0.0249$ and $\lambda_{2}=-40.1$. The response of this loading path is given in Figure 8. Along this loading path, three instability cones have been computed, the first one before reaching the $\sigma_{1}-\lambda_{1} \sigma_{2}-\lambda_{2} \sigma_{3}$ peak, the second one at the peak and the third one after the peak. Figure 9 presents this path with the cones in the 3D strain space.

Through Figures 9 and 10, the following comments can be made. Before reaching the first cone, the loading path is outside the bifurcation domain, no bifurcation and failure can occur. At the first cone, the loading path just come in the bifurcation domain. Nevertheless, loading path is outside the cone, as a consequence no instability can occur at this state along this path. At the second cone, the loading path goes through the peak of $\sigma_{1}-\lambda_{1} \sigma_{2}+\lambda_{1} \lambda_{2} \sigma_{3}$, as a consequence $w_{2}$ vanishes and the loading path is just tangent to the instability cone. The path becomes unstable. Effective failure occurs if $\sigma_{1}-\lambda_{1} \sigma_{2}+\lambda_{1} \lambda_{2} \sigma_{3}$ is driven. In our case, $\varepsilon_{1}$ is driven, then the peak can be dropped in. After the peak, $w_{2}<0$, then the loading path is unstable. At the third cone, path is clearly inside the cone. It is worth noting that all stress-strain states presented are inside the plasticity limit. Therefore, concept of limit state and flow rule is generalized here for stress-strain states situated inside the bifurcation domain but strictly before the plasticity limit condition.

The simulation of failure with the discrete element method presented in Section 2.2, was done in axisymmetric conditions. However conclusions given here in 

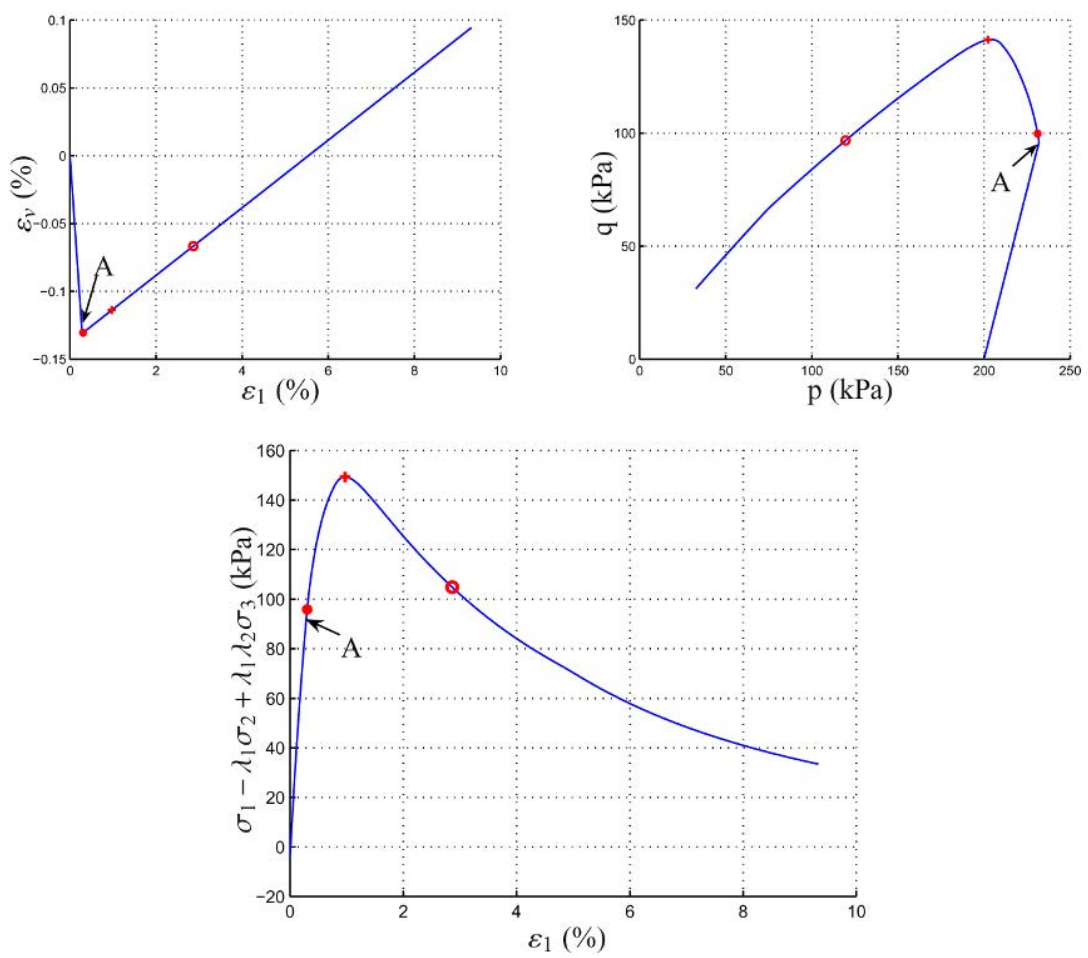

Fig. 8 Response to the drained triaxial path until $\sigma_{1}=298 \mathrm{kPa}, \sigma_{2}=\sigma_{3}=200 \mathrm{kPa}, \varepsilon_{1}=0.292 \%$ and $\varepsilon_{2}=\varepsilon_{3}=-0.079 \%$ (point A); then response to the proportional strain path (Eq. 24) defined by $\lambda_{1}=0.0249$ and $\lambda_{2}=-40.1$

three-dimensional conditions hold in axisymmetric conditions. Hence, in Figure 1 failure occurs at the peak of $\sigma_{1}-\lambda \sigma_{3}$, because, even if the loading path is inside the bifurcation domain before this peak, the strain direction defined by the loading program is included in the cone of unstable strain directions, for the first time, at the peak of $\sigma_{1}-\lambda \sigma_{3}$.

\section{From Limit States to Failure Occurrence}

The objective of this section is to confirm through DEM simulations, that inside the bifurcation domain displayed in Section 3.2.2, the occurrence of failure requires: the loading directions to belong to the cone of unstable loading directions (associated to the current stress state); and the control loading parameters to be mixed (involving strain and stress terms). 


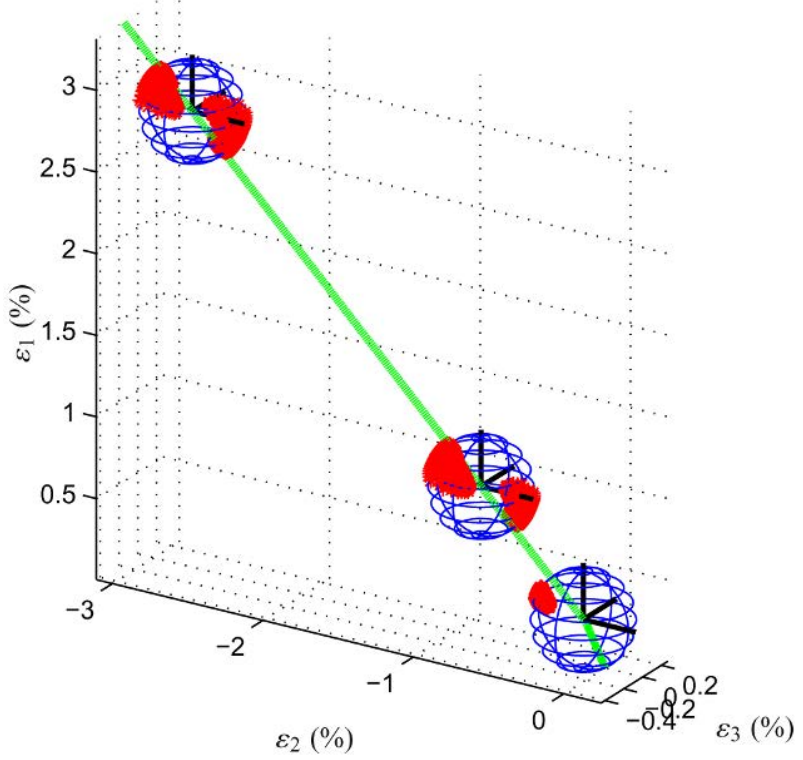

Fig. 9 Loading path in the 3D strain space with three instability cones computed before, at, and after the $\sigma_{1}-\lambda_{1} \sigma_{2}-\lambda_{2} \sigma_{3}$ peak

\subsection{Mixed Loading Parameters}

Concerning the control mode of the loadings, stress probes presented in Section 3 were conducted with only stress control parameters $\left(\delta \sigma_{1}=c s t 1\right.$ and $\delta \sigma_{3}=c s t 3$, with constants $\operatorname{cst} 1$ and $\operatorname{cst} 3$ chosen to impose the desired direction $\alpha$ ). For such full stress controls, no failure can occur before the plastic limit condition [12], representing limit stress states. That is why we have been able to simulate these stress probes, where numerical samples recovered an equilibrium state after each probe direction, whatever the sign of the second-order work computed.

Let us now reconsider these stress probes as proportional stress loading paths where the ratio between $\delta \sigma_{1}$ and $\delta \sigma_{3}$ can be defined by:

$$
\delta \sigma_{1}-\lambda \delta \sigma_{3}=0 \quad \text { where: } \lambda=\sqrt{2} \sin \alpha / \cos \alpha \quad \text { for } \alpha \in[180 \mathrm{deg} ; 270 \mathrm{deg}[
$$

The energy conjugated variables are thus $\delta \sigma_{1}-\lambda \delta \sigma_{3}=0$ and $\delta \varepsilon_{1}$ on one hand, and $\delta \sigma_{3}$ and $\lambda \delta \varepsilon_{1}+2 \delta \varepsilon_{3}$ on the other hand. For $\lambda=1$, the stress direction is $\alpha=215.3 \mathrm{deg}$ and corresponds to a path where the deviatoric stress is constant $\delta q=0$; in addition, $\delta \varepsilon_{1}+2 \delta \varepsilon_{3}=\delta \varepsilon_{v}$. For sample $E 3$, this latter stress path is included in the cone of instability for $\eta=0.46$, as displayed in Figure 11. Figure 12 shows for this stress probe direction, totally controlled with stress parameters, that there is no outburst of kinetic energy as expected, and that the change of the incremental volumetric strain $\delta \varepsilon_{v}$ presents a minimum (maximum of dilatancy). This 

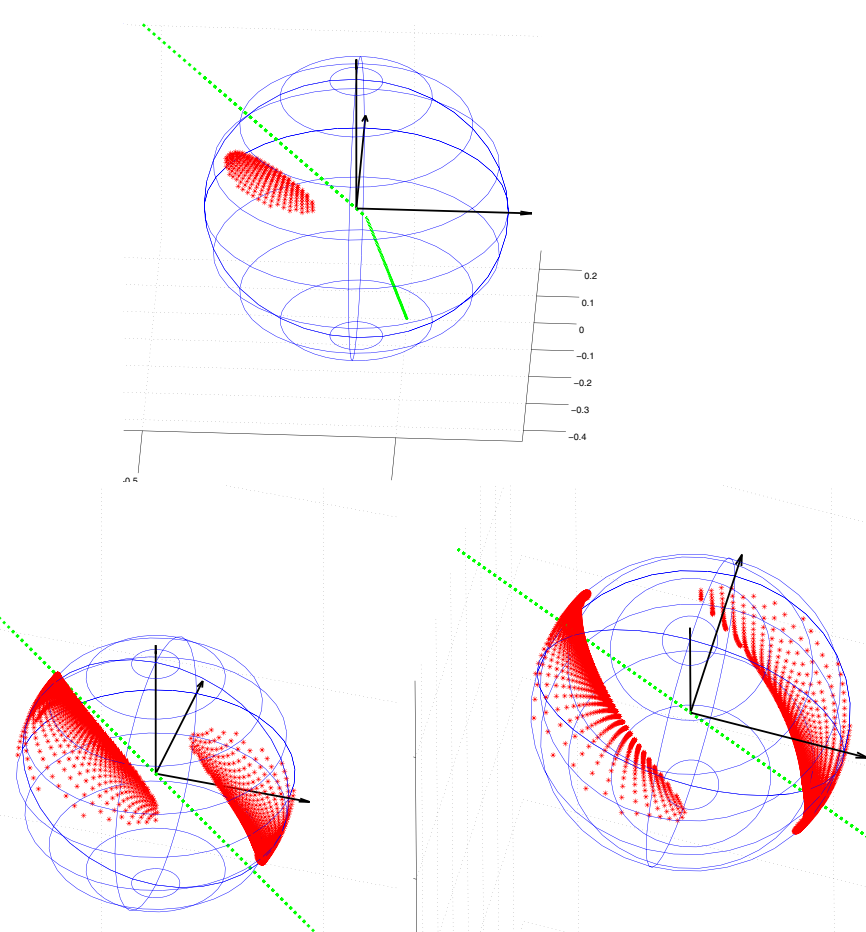

Fig. 10 Zoom of the three cones of Figure 9

Fig. 11 Comparison of the normalized second-order work between samples $E 1$ and $E 3$, for the confinement pressure $\sigma_{3}=100 \mathrm{kPa}$

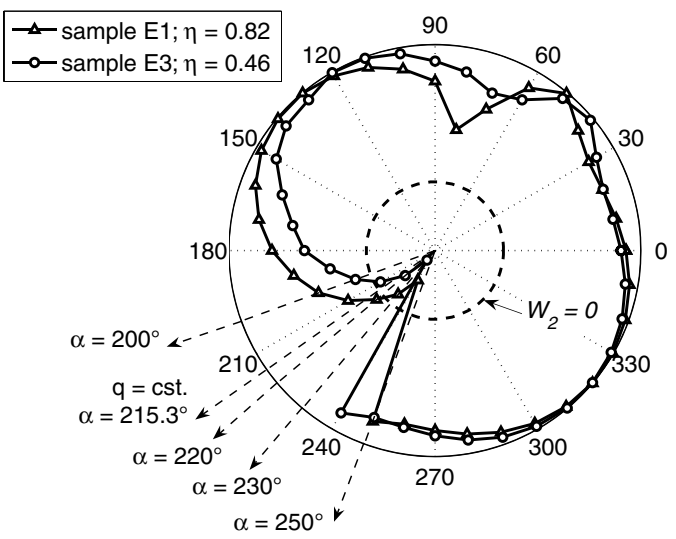

extremum of volumetric strain corresponds to a limit state. The proportional stress path characterized by $\lambda=1$ can also be followed by controlling the sample with 

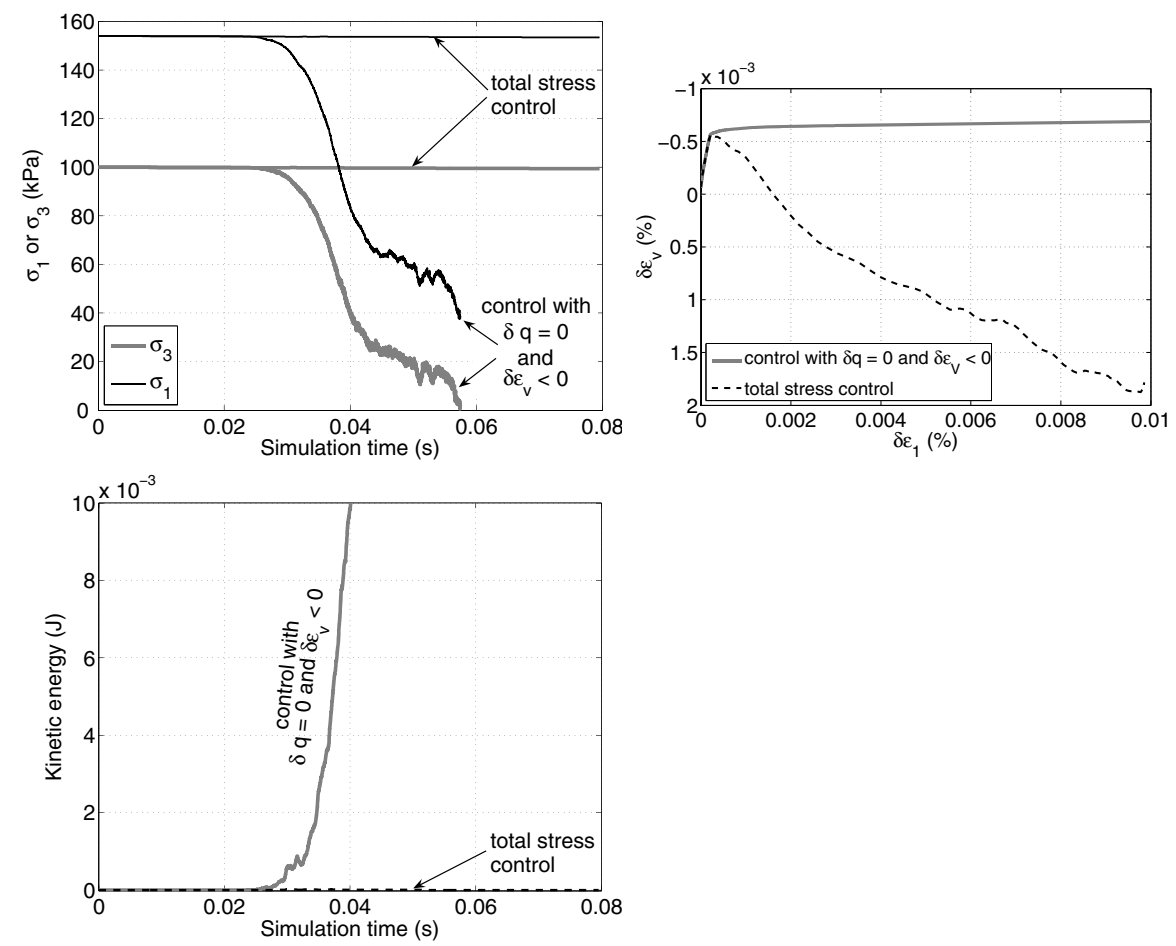

Fig. 12 Comparison of responses of sample $E 3$ along a constant deviatoric stress direction, controlled either with stress parameters only or with mixed (stress and strain) parameters

mixed control parameters ${ }^{4}$ defined by

$$
\delta q=0 \quad \text { and } \quad \delta \varepsilon_{v}=\text { negative const. }
$$

Hence, a constant dilatancy rate is imposed. Response of the sample is presented in Figure 12. Until the minimum of $\delta \varepsilon_{v}$, the kinetic energy stays low (quasi-static response) and the sample followed the imposed path. Then, when the limit state constituted by the minimum of $\delta \varepsilon_{v}$ is reached, this latter cannot be exceeded, stresses vanish suddenly ${ }^{5}$ and an outburst of kinetic energy occurs highlighting the transition to a dynamic response. This simulated response corresponds to a proper failure that can be seen as a sudden liquefaction. Consequently, failure of the sample has been triggered by the mixed mode of control, whereas there was no characteristic feature of failure for a total stress control.

\footnotetext{
${ }^{4}$ To ensure such a mixed control mode (in stress and strain) with control parameters defined as linear combinations of principal stress or strain components, a specific algorithm is run every time step of the DEM cycle; details can be found in $[17,20]$.

${ }^{5}$ Due to the dynamic response of the sample, stress components do not vanish all together.
} 
Table 2 Stress directions and belonging to cones of instability for samples $E 1$ and $E 3$

\begin{tabular}{llll}
\hline$\alpha$ & $\lambda$ & \multicolumn{2}{c}{$\in$ cone of unstable stress directions ? } \\
$(\mathrm{deg})$ & & sample $E 1(\eta=0.82)$ & sample $E 3(\eta=0.46)$ \\
\hline 200 & 0.515 & No & No \\
220 & 1.19 & No & Yes \\
230 & 1.69 & Yes & Yes (close to the limit of the cone) \\
250 & 3.89 & No & No \\
\hline
\end{tabular}

\subsection{Stable and Unstable Loading Directions}

In Section 4.1 only one stress loading direction, belonging to the cone of instability for sample $E 3$, has been considered. We investigate now a wider range of stress directions to compare responses of samples $E 1$ and $E 3$ along each direction, and in particular for directions included in cones of instability and for others excluded. Figure 11 displays the normalized second-order work computed at $\eta=0.82$ for sample $E 1$ and $\eta=0.46$ for $E 3$. The two cones of instability, corresponding to the two samples, are not superimposed. For the loosest sample E3, the cone is more opened and includes lower values of $\alpha$ than for the densest sample $E 1$ [20]. As shown by Darve et al. [4], this difference is typically related to the difference in porosity of the two samples.

In the following four stress directions are considered $(\alpha=200,220,230$ and $250 \mathrm{deg}$ ), their belonging to cones of instability is summarized in Table 2 . To be able to trigger the failure along these stress directions, mixed loading control parameters are chosen as detailed in Section 4.1. $\delta \sigma_{1}-\lambda \delta \sigma_{3}=0$ is imposed to prescribe the desired stress direction (see Table 2 for values of $\lambda$ ). However, instead of imposing a change of the parameter $\lambda \delta \varepsilon_{1}+2 \delta \varepsilon_{3}$, the latter is kept constant, i.e. $\lambda \delta \varepsilon_{1}+$ $2 \delta \varepsilon_{3}=0$ (see footnote 4 ). If simulations are run in these conditions there is no evolution of samples, they stay at their initial mechanical state maintained by these two control parameters. A perturbation is necessary to conclude about the stability of the mechanical state considered with the control parameter chosen.

Since simulations are performed without gravity, some particles float in the pores of samples and are not involved in the contact force network, at the equilibrium state considered. The sample is perturbed by imposing an instantaneous velocity in a random direction on eight floating particles. Samples are virtually split into eight sub-parallelepipeds, each perturbed particle is chosen randomly in each subparallelepiped respectively. The velocity imposed to each particle is computed such that the value of kinetic energy provided is equal for each particle. The perturbation corresponds to a total external input of kinetic energy of $10^{-5} \mathrm{~J}$. This input is small compared with the maximum value of kinetic energy "naturally" developed for fully stress controlled probes: $10^{-4} \mathrm{~J}$. It is worth noting that responses simulated with such a perturbation are totally similar to responses that would be obtained by imposing a change of the control parameter involving strain terms, i.e. $\lambda \delta \varepsilon_{1}+2 \delta \varepsilon_{3}<0$, 
$\alpha=200 \mathrm{deg} ; \lambda=0.515$
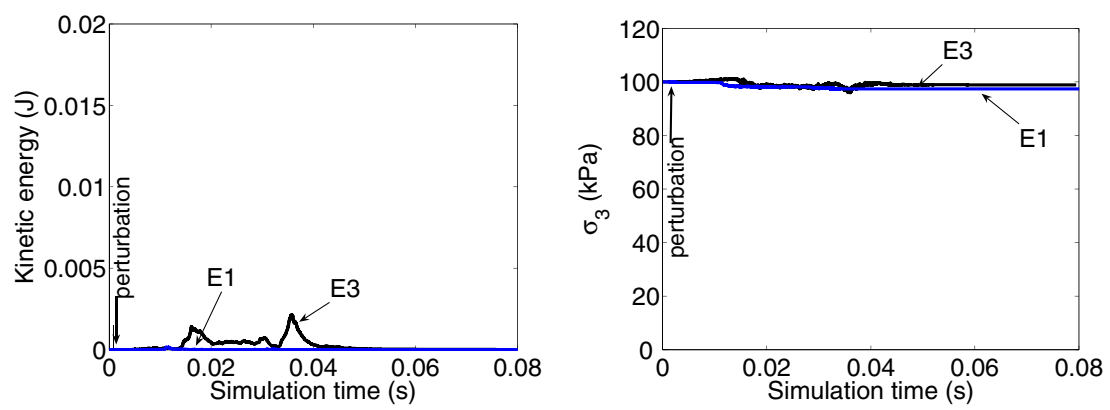

$\alpha=220 \mathrm{deg} ; \lambda=1.19$
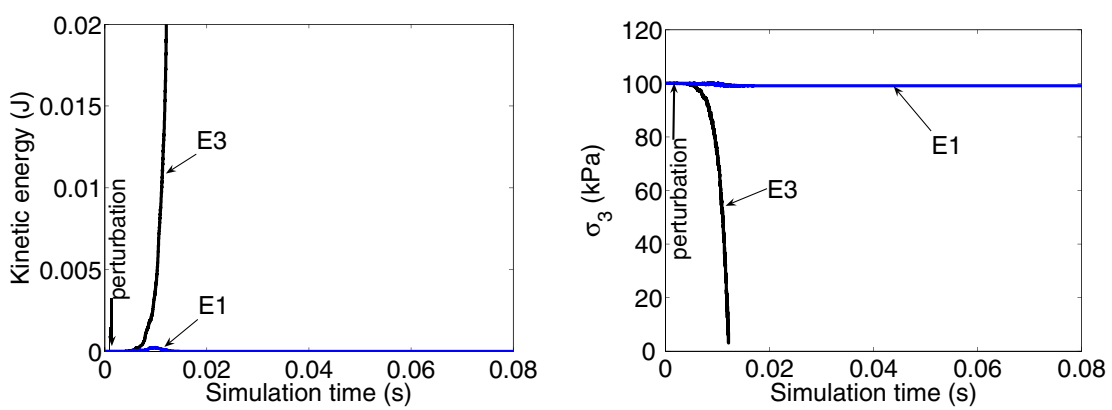

Fig. 13 Responses of samples $E 1$ and $E 3$ to a perturbation in kinetic energy, for the stress directions $\alpha=200$ and $220 \mathrm{deg}$

as shown in $[17,18]$ (see also [5] for proportional strain loading paths). It is simply another way to test the stability of samples.

Figures 13 and 14 present the simulated responses of samples $E 1$ and $E 3$ in terms of time evolutions of the kinetic energy and of radial stress $\sigma_{3}$. Changes of the axial stress $\sigma_{1}$ are very close to $\sigma_{3}$ changes, and are thus not displayed. Arrows in diagrams indicate the time of application of the perturbation.

- Stress directions $\alpha=200$ and 250 deg are not included in cones of instability for both samples. For these directions, after the application of the perturbation, the kinetic energy fluctuates slightly but finally vanishes, and both samples recover an equilibrium at a stress state close to the initial one.

- The stress direction $\alpha=220 \mathrm{deg}$ is included in the cone of instability for the sample $E 3$ but not for the sample $E 1$. The failure, highlighted by the outburst of kinetic energy and the vanishing of stresses, is observed only for $E 3$, whereas $E 1$ is almost unaffected by the perturbation.

- The stress direction $\alpha=230 \mathrm{deg}$ is included in the cone of instability of both samples $E 1$ and $E 3$. For both samples failure occurs as shown by the sudden vanishing of stresses. However for $E 3$ the burst of kinetic energy has not com- 
$\alpha=230 \mathrm{deg} ; \lambda=1.69$
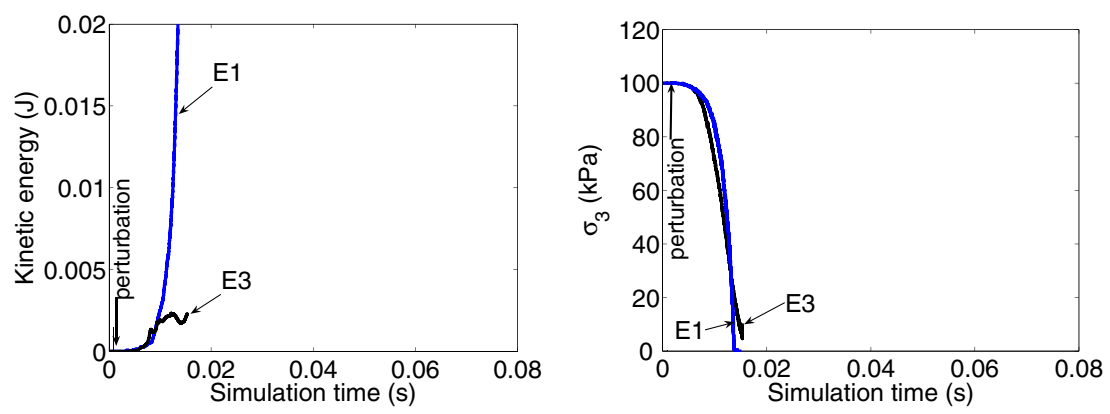

$\alpha=250 \mathrm{deg} ; \lambda=3.89$
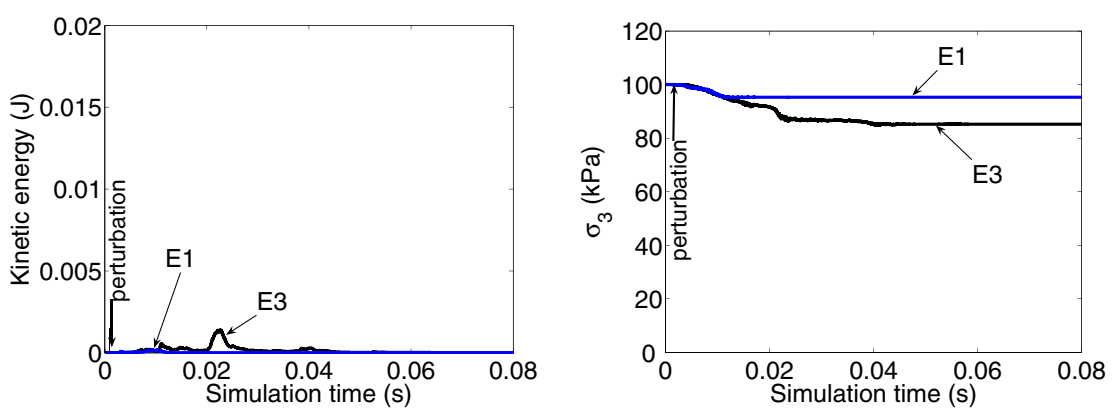

Fig. 14 Responses of samples $E 1$ and $E 3$ to a perturbation in kinetic energy, for the stress directions $\alpha=230$ and $250 \mathrm{deg}$

pletely developed. This intermediate result can be explained by the proximity of the tested direction, $\alpha=230 \mathrm{deg}$, to the boundary of the cone.

These results show clearly that, even if the mechanical state of the sample is controlled with mixed parameters (as defined in Section 4.1), failure occurs only when the loading direction is included in the cone of instability. In other words, failure can be triggered only if the loading direction is associated with a nil or negative value of the second-order work.

\section{Conclusion}

Three approaches have been considered in this chapter to investigate what is failure of granular media samples: theoretical considerations, application of phenomenological rate-independent constitutive relations, and - in a more detailed manner simulations of failure by a discrete element model. 
1. The theoretical developments have provided a firm basis to explain the link between second-order work and failure. Indeed when the second-order work is taking a zero (or negative) value in a given stress direction, if a proper arbitrarily small additional load is applied in this direction, a burst of kinetic energy appears leading to a dynamic regime of deformations, which is typical of failure.

2. The 3D phenomenological analysis has leaded to the existence of a bifurcation domain, preserving the conical structure of Mohr-Coulomb criterion, and of instability cones, which can be not unique and have an elliptical cut in 3D.

3. The discrete element method is able to describe failure in a very natural way without any ad-hoc ingredient. This is the essential reason why this method has been extensively used to investigate failure in granular media. Bifurcation domain and instability cones have been obtained as conjectured by the elastoplastic theory.

Moreover, the three necessary and sufficient conditions for an effective failure have been successfully checked:

- the stress state has to belong to the bifurcation domain,

- the actual stress direction has to be inside an instability cone,

- the proper loading parameters have necessary to be mixed ones.

The observed failure modes are characterized by bursts of kinetic energy, exponentially growing strains, and decreasing stresses. This is basically a kind of generalized liquefaction. Because no strain localization pattern has been observed (and moreover the strain localization criterion is not satisfied at these stress-strain states), these failure modes have been called "diffuse" [4].

These various features and conclusions have been also observed in some laboratory experiments performed in drained and undrained conditions on Hostun sand [5].

\section{References}

1. Cundall, P.A., Strack, O.D.L., A discrete numerical model for granular assemblies. Geotechnique, 29(1):47-65, 1979.

2. Darve, F., Flavigny, E., Meghachou, M., Yield surfaces and principle of superposition revisited by incrementally non-linear constitutive relations. International Journal of Plasticity, 11(8):927-948, 1995.

3. Darve, F., Labanieh, S., Incremental constitutive law for sands and clays. simulations of monotonic and cyclic tests. International Journal for Numerical and Analytical Methods in Geomechanics, 6:243-275, 1982.

4. Darve, F., Servant, G., Laouafa, F., Khoa, H.D.V., Failure in geomaterials: Continuous and discrete analyses. Computer Methods in Applied Mechanical Engineering, 193(27-29):30573085, 2004.

5. Darve, F., Sibille, L., Daouadji, A., Nicot, F., Bifurcations in granular media: Macro- and micro-mechanics approaches. Comptes Rendus Mécanique, 335(9-10):496-515, 2007.

6. Desrues, J., Shear band initiation in granular materials: Experimentation and theory. In Geomaterials Constitutive Equations and Modelling, F. Darve (ed.), pp. 283-310. Elsevier, Taylor and Fancis Books, 1990. 
7. Donzé, F.V., Magnier, S.A. Spherical discrete element code. In Discrete Element Project Report 2, Laboratory GEOTOP, Université du Québec à Montréal, 1997.

8. Gudehus, G., A comparison of some constitutive laws for soils under radially symmetric loading and unloading. In Proceedings 3rd Numererical Methods in Geomechanics, Aachen, 2-6 April. A.A. Balkema, Rottedam, 1979.

9. Hill, R., A general theory of uniqueness and stability in elastic-plastic solids. Journal of the Mechanics and Physics of Solids, 6:239-249, 1958.

10. Nicot, F., Darve, F., A micro-mechanical investigation of bifurcation in granular materials. International Journal of Solids and Structures, 44:6630-6652, 2007.

11. Nicot, F., Darve, F., Khoa, H.D.V., Bifurcation and second order-work in granular materials. International Journal for Numerical and Analytical Methods in Geomechanics, 31:10071032, 2007.

12. Nova, R., Controllability of the incremental response of soil specimens subjected to arbitrary loading programmes. J. Mech. Behav. Mater., 5(2):193-201, 1994.

13. Prunier, F., Laouafa, F., Darve, F., 3D bifurcation analysis in geomaterials, investigation of the second order work criterion. European Journal of Environmental and Civil Engineering, 13(2):135-147, 2009.

14. Prunier, F., Laouafa, F., Lignon, S., Darve, F., Bifurcation modeling in geomaterials: From the second-order work criterion to spectral analyses. International Journal for Numerical and Analytical Methods in Geomechanics, 33:1169-1202, 2009.

15. Prunier, F., Nicot, F., Darve, F., Laouafa, F., Lignon, S., 3D multi scale bifurcation analysis of granular media. Journal of Engineering Mechanics (ASCE), 135(6):493-509, 2009.

16. Rice, J.R., The localization of plastic deformation. In Theoretical and Applied Mechanics, W.T. Koiter (ed.), pp. 207-220. North-Holland Publishing Compagny, Delft, 1976.

17. Sibille, L., Modélisations discrètes de la rupture dans les milieux granulaires. $\mathrm{PhD}$ Thesis, Institut National Polytechnique de Grenoble, 2006.

18. Sibille, L., Donzé, F.V., Nicot, F., Chareyre, B., Darve, F., From bifurcation to failure in a granular material, a DEM analysis. Acta Geotechnica, 3(1):15-24, 2008.

19. Sibille, L., Nicot, F., Donzé, F.V., Darve, F., Material instability in granular assemblies from fundamentally different models. International Journal for Numerical and Analytical Methods in Geomechanics, 31:457-481, 2007.

20. Sibille, L., Nicot, F., Donzé, F.V., Darve, F., Analysis of failure occurrence from direct simulations. European Journal of Environmental and Civil Engineering, 13:187-201, 2009. 